\title{
Developing the theory of working memory
}

\author{
JOHN T. E. RICHARDSON \\ Brunel University, Uxbridge, Middlesex, England
}

\begin{abstract}
The theory of working memory was devised to explain the effects of a concurrent memory load in various experimental situations in terms of the operation of a central executive processor and a phonemic response buffer. It also explains the effects of phonemic similarity, articulatory suppression, word length, and unattended speech. Experiment 1 demonstrated that a concurrent memory load markedly reduced the phonemic similarity effect in immediate serial recall, which was taken to support the concept of a limited-capacity phonemic response buffer. A more detailed analysis of the results suggested that a concurrent memory load may affect the storage capacity of the central executive processor and the translation of orthographic stimuli into phonological representations, as well as the storage capacity of the phonemic response buffer. Experiment 2 showed that a concurrent freerecall task reduced the phonemic similarity effect in immediate serial recall, but only in the case of visually presented sequences of items. Moreover, unattended speech was found to have no effect upon performance in immediate free recall. These results were taken to imply that the phonemic response buffer contributes only to performance in cognitive tasks that require the accurate retention of serial-order information.
\end{abstract}

A series of experiments reported by Baddeley and Hitch (1974) investigated the effects of a concurrent serial-recall task upon performance in tests of reasoning, comprehension, and free recall. Such a "concurrent memory load" was expected to "absorb some of the storage capacity of a limited capacity working memory system" (p. 50), and thus to impair performance in any criterion task that relied upon such a system. Baddeley and Hitch found that a concurrent memory load of six items reduced performance in all three sorts of task. They also showed that phonemic similarity among the stimulus items impaired both reasoning and comprehension, and that suppressing any relevant articulatory activity by requiring the subjects to produce irrelevant vocalizations impaired performance in free recall and, to a lesser extent, in reasoning. These results were taken to support the notion of working memory as a short-term informationstorage system that had access to phonemic coding.

Such a notion was entirely compatible with the prevalent information-processing approach to human cognition, and in itself involved no radical departure from previous theoretical accounts of short-term mem-

A presentation on which this paper is based was given to an International Conference on "Memory" organized by the Cognitive Psychology Section of the British Psychological Society at Plymouth Polytechnic on September 9, 1981. I am indebted to Alan Baddeley, Graham Hitch, Stephen Monsell, and two anonymous reviewers for their comments, advice, and encouragement, and also to colleagues and students of Brunel University and the Open University. I am grateful to Shareen Courtney, Tom Marden, and Nick Abbot for their assistance in running Experiment 1, and to Irene Short, Janet Pearson, and Linda Burman for their assistance in running Experiment 2. My mailing address is: Department of Psychology, Brunel University, Uxbridge, Middlesex UB8 3PH, England. ory. In fact, it is now widely accepted that the span of immediate memory is determined by the available storage capacity of working memory, conceived as a general information-processing system. Nevertheless, Klapp, Marshburn, and Lester (1983) pointed out that it is difficult to reconcile this assumption with the rather poor correlations that are typically found between digit span and full-scale general intelligence, or with the existence of neurological patients who demonstrate a selective impairment of verbal short-term memory in the absence of any wider impairment of overall cognitive abilities. These findings entail that there exists a limitedcapacity memory component that is not involved in general cognitive processing. Klapp et al. reported a series of experimental investigations (to be mentioned later in this paper) that cast further doubt upon the existence of a unitary mechanism of working memory that has both processing and storage functions in human cognition.

A more viable alternative is to provide an articulated theory of working memory as a system of interrelated mechanisms that are differentially responsible for these functions. In fact, Baddeley and Hitch (1974) elaborated their account by distinguishing between a relatively active, flexible, central executive processor and an optionally employed, although relatively passive, phonemic response buffer. The former was assumed to be responsible for information processing and decision making, whereas the latter was regarded as a slave system that "is able to store a limited amount of speechlike material in the appropriate serial order" (see also Baddeley, 1976, p. 176, and Baddeley \& Lewis, 1981). In other words, although the properties of the central executive processor were left somewhat unclear, the phonemic response buffer was a distinctive hypothetical 
entity in two important respects: First, the characteristic form of encoding employed by this component was assumed to be "speech-like" or phonemic in nature; second, the characteristic form of organization employed by this component was assumed to be temporal and serial.

The accounts of this theory that were variously given by Baddeley $(1976,1979)$, Baddeley and Hitch (1974), Baddeley, Thomson, and Buchanan (1975), and Hitch (1980) suggested that it was merely a framework within which one might conceptualize different experimental tasks and formulate various theoretical questions. However, Baddeley (1981) proposed that the future usefulness of the concept of working memory would depend upon the development and empirical evaluation of specific models of working memory that would allow testable and nontrivial predictions. Two fundamental issues that appear to be open to empirical investigation are: whether the component of the informationprocessing system that employs phonemic coding is also responsible, in whole or in part, for limitations of the capacity of immediate memory; and whether the same component is employed solely for the retention of serial-order information. These issues were explored in the two experiments reported in this paper. First, however, it is useful to consider the various experimental procedures that are taken to be alternative, convergent indicators of the operation of the phonemic response buffer.

\section{Word-Length Effect}

Baddeley and Hitch (1974) postulated the phonemic response buffer as a "subcomponent or slave system of working memory that can maintain phonemically encodable material through subvocal rehearsal" (Baddeley \& Lewis, 1981, p. 211). However, they were fairly agnostic as to the exact nature of the encoding employed, and did not specify whether information was assumed to be stored in the form of acoustic traces, articulatory programs, or more abstract, phonemic representations. Certainly, it is well known that the effects of phonemic similarity in short-term recall are open to a variety of theoretical interpretations.

Subsequently, Baddeley et al. (1975) found that performance in short-term serial recall on a given vocabulary of stimulus material was negatively correlated with the time taken to articulate that material, even when the number of syllables and the number of phonemes that the material contained were held constant. This wordlength effect was obtained even when the material was presented visually, although not when the subjects were required to produce irrelevant vocalizations, and a further experiment showed that reducing the temporal duration of auditory stimuli had no effect upon performance (Hitch \& Baddeley, 1977, p. 102). The latter results appeared to rule out any possible explanation of the word-length effect in terms of an auditory memory system.
Since articulation latency depends not only upon the number of phonemes in a word, but also upon the nature of those phonemes (such as whether vowels are long or short), these results can be accommodated within an account based upon abstract, phonemic encoding if it is assumed that irrelevant vocalizations inhibit such encoding. However, Baddeley et al. (1975) attributed the word-length effect to the mechanisms underlying speech production. It was suggested that the response buffer "resembles a tape recorder, whose capacity is limited by time rather than by the amount of information or number of events" (Baddeley, 1976, p. 178), or that it "functions rather as a tape loop of limited duration" (Baddeley, 1979, p. 357); and the original phonemic response buffer was subsequently referred to as an "articulatory rehearsal loop," an output buffer containing stored speech responses.

\section{Effects of Articulatory Suppression}

Baddeley $(1976$, pp. 178; 180) suggested that the use of an articulatory store would be preempted by requiring the subjects to produce irrelevant vocalizations, a procedure usually known as "articulatory suppression." If such a mechanism is intended to explain the effects of phonemic similarity and word length in immediate serial recall, then it follows that articulatory suppression should abolish those effects. Under conditions of visual presentation, irrelevant vocalizations do indeed eliminate both the phonemic similarity effect (Richardson, Greaves, \& Smith, 1980) and the word-length effect (Baddeley et al., 1975). However, under conditions of auditory presentation, irrelevant vocalizations do not remove either the effect of phonemic similarity (Levy, 1971; Murray, 1968; Peterson \& Johnson, 1971) or the effect of word length (Baddeley et al., 1975). These results have three major implications for the theory of working memory:

1. Articulatory suppression appears to inhibit the phonological encoding of visually presented material, but not that of auditorily presented material. In other words, irrelevant vocalizations have a selective effect upon the translation of orthographic stimuli into a phonological representation in working memory (Baddeley et al., 1975; Hitch \& Baddeley, 1977, p. 104; Salamé \& Baddeley, 1982). This seems to be a specific function of the central executive processor. When subjects are required to retain an arbitrary sequence of visually presented items without the use of phonemic encoding, there is a residual level of retention that presumably reflects the capacity of the central executive processor to maintain a purely visual representation of the sequence (see Klapp et al., 1983). Although there is good evidence for the persistence of such representations in shortterm memory (e.g., Baddeley, 1976, pp. 204-211), their value in conventional verbal-learning tasks appears to be extremely limited (Hiles, 1974; Warrington \& Shallice, 1972).

2. Articulatory suppression does not appear to inter- 
fere with the actual maintenance of phonemically encoded information in working memory. This implies that the articulatory system is not in itself responsible for the effects of phonemic coding (Hitch \& Baddeley, 1977 , p. 103). It appears that the word-length effect is attenuated under conditions of auditory presentation when irrelevant vocalizations are required during both presentation and recall (Baddeley, 1981). This suggests that articulatory suppression may prohibit the retrieval of information from the phonemic response buffer.

3. Phonological information can be represented in working memory under conditions of auditory presentation even in the absence of relevant subvocal articulation. This suggests that the phonemic response buffer can be primed either by articulation or by acoustic stimulation (Baddeley, 1979, 1981).

Klapp et al. (1983) also argued against the concept of an articulatory rehearsal loop on the basis of their finding that simultaneous auditory presentation was more effective than silent articulation in supporting the retention of visually presented sequences of digits. They concluded that "one component of span memory is auditory rather than articulatory" (p. 246). However, there are at least two problems with this conclusion. First, the basic finding may create some difficulty for the idea of an articulatory store, but it does not permit one to discriminate between acoustic traces and more abstract, phonemic representations. Indeed, it may be taken as evidence that the latter representations are generated more readily on the basis of auditory input than on the basis of articulatory information. Second, the procedure adopted by Klapp et al. failed to control for the additional processing load involved in silent articulation of a digit sequence. The observed variation in performance can thus be attributed to merely the cognitive demands of digit naming. Klapp et al. went on to show that a concurrent memory load would impair performance in simple reasoning tasks, but not if an unfilled interval of $6.5 \mathrm{sec}$ was interpolated between the presentation of the sequence used as a memory load and the presentation of the reasoning problem. This suggests that central processing capacity is employed in the encoding of visually presented sequences into the phonemic response buffer, but not in the retention or maintenance of encoded material within the phonemic response buffer.

\section{Effects of Unattended Speech}

The idea that the phonemic response buffer might be primed by either acoustic or articulatory stimulation was developed in a recent series of experiments by Salamé and Baddeley (1982). They noted that irrelevant, unattended speech impaired the immediate serial recall of visually presented sequences of digits. The magnitude of the impairment was influenced by the phonemic similarity between the irrelevant words and the material to be remembered, but not by semantic characteristics of the unattended speech. This suggested that the irrelevant material was disrupting the contribution of a phonemically based, nonlexical memory system. Articulatory suppression was found to remove the disrupting effect of unattended speech, and a previous experiment by Colle and Welsh (1976) had found that unattended speech impaired the recall of phonemically distinct sequences but not that of phonemically confusable sequences. These results tended to identify the relevant memory system with the phonemic response buffer. However, long words were no more disruptive as irrelevant speech stimuli than were short words, which Salamé and Baddeley took to mean that unattended speech stimuli were not themselves articulated, but were encoded directly into a phonological memory system. In short, the most recent statement of the workingmemory model specifies an abstract phonological component that is accessible either through an obligatory representation of auditory stimuli or through an optional process of subvocal articulation.

\section{EXPERIMENT 1}

The original paper by Baddeley and Hitch (1974) suggested that the phonemic response buffer "plays a major role in determining the occurrence of ... acoustic similarity effects in memory" (p. 79). Subsequently, Baddeley $(1976$, pp. 178; 180) was quite adamant that the effects of phonemic similarity, articulatory suppression, and word length were to be ascribed to the functioning of the phonemic response buffer. The effects of phonemic similarity and of articulatory suppression were attributed to its use of speech-based encoding, whereas the effect of word length was attributed to its limited capacity. Although recent research has prompted some revision of the original arguments, it is still possible to hold that the hypothesized phonemic response buffer is the locus of effects that are attributable to the use of phonemic coding in short-term memory.

As Baddeley et al. (1975) pointed out, this implies that the central executive processor does not itself have access to phonemic coding (although it may well have access to a variety of other memory codes). There is obvious theoretical elegance in postulating a single component to explain several different empirical phenomena in this manner (Baddeley \& Lewis, 1981), and it has proved to be a useful concept in investigating developmental and cross-cultural variations in memory span (Ellis \& Hennelly, 1980; Nicholson, 1981). There is apparent counterevidence in the finding that articulatory suppression has little effect upon either the speed or the accuracy with which subjects judge whether or not visually presented pairs of words rhyme (Baddeley \& Lewis, 1981), but this is generally taken to imply that the system responsible for accessing prelexical or "input" phonology in reading is functionally distinct from the system that is responsible for effects of phonemic coding in short-term memory (Baddeley, Eldridge, \& Lewis, 1981; Besner, Davies, \& Daniels, 1981; Coltheart, 1980). In support of this, Besner (1982) 
recently showed that the advantage of pseudohomophones (that is, nonwords that are homophonic to words) over other pronounceable nonwords in short-term recall is not removed by articulatory suppression.

Nevertheless, it is assumed in the standard account of the phonemic response buffer that it is responsible both for the effects of phonemic coding in short-term memory and, at least in part, for effects that are attributable to limitations of storage capacity (such as the effect of word length). This assumption leads to a specific prediction of an interaction between the effect of a concurrent memory load and the effect of phonemic similarity in immediate serial recall. On the one hand, it would be predicted that a concurrent memory load would occupy some of the storage capacity of the hypothesized short-term phonemic store. The contribution of that store to performance in immediate serial recall should therefore be reduced, which would attenuate the overall effect of phonemic similarity. On the other hand, it would be predicted that phonemic similarity among the stimulus items would reduce the efficacy of a limited-capacity store that was based upon phonological representations. Once again, the contribution of such a store to performance in immediate serial recall should therefore be reduced, and this would attenuate the effect of a concurrent memory load.

Experiment 1 tested these proposals by manipulating the presence or absence of a concurrent memory load and the phonemic confusability of the stimulus material in an orthogonal manner. One of the experiments reported by Klapp et al. (1983) demonstrated that performance in serial recall would be disrupted by an interpolated digit-span task, but the phonemic confusability of the stimulus material was not manipulated. In Experiment 1, sequences of consonants and words were presented simultaneously, and both sets of material had to be remembered in the order of presentation. The design of this experiment was therefore symmetri$\mathrm{cal}$, in that each of the two sequences of items presented on a given trial constituted a concurrent memory load for the other, and it was anticipated that both types of material would show the predicted interaction between the two major variables.

\section{Method \\ Design. The subjects were presented with sequences of letters and words simultaneously. The phonological confusability of the letters, the phonological confusability of the words, and the type of material to be recalled were orthogonally varied, following a within-subjects design, in an unpredictable fashion across differ- ent trials. For half of the subjects, the sequence to be recalled on each trial was identified in advance, and it may be assumed that they were learning this material with no concurrent memory load. For the other half of the subjects, the criterion task was not specified in advance, and it may be assumed that the se- quences presented simultaneously with the material to be re- called constituted a concurrent memory load. Independently of this, written recall was used for half of the subjects, and oral recall was used for the remainder.}

Subjects. This experiment involved 20 undergraduate students at Brunel University who were taking an introductory course in laboratory methods and 20 students applying for admission to the Psychology Honours Degree course who volunteered to take part.

Materials. The four sets of stimulus materials were taken from Experiments V and VI reported by Baddeley (1968): One set contained six phonemically confusable letters $(b, c, d, p, t$, $v)$; another set contained six phonemically distinct letters $(j, k$, $1, r, w, y) ;$ a third set contained six phonemically confusable words (cad, can, cap, cat, mad, man); and the fourth set contained six phonemically distinct words matched for frequency with those in the previous set (bar, bun, day, few, rig, sup). Twenty random orderings of each of the four sets of stimuli were produced. The sequences were assigned in pairs at random to 40 trials, with the constraint that two of every successive set of eight trials combined each of the sets of letters with each of the sets of words; one of these trials was randomly assigned for letter recall, and the other for word recall.

Procedure. The stimulus material was initially displayed on the output scope of a computer; the letters were presented above the words and simultaneously with them, at a rate of $1 \mathrm{item} / \mathrm{sec}$ and in uppercase. Each trial was preceded by a sequence of warning signals, and was followed by the appropriate recall signal, "RECALL LETTERS" or "RECALL WORDS." The latter signal was presented for $20 \mathrm{sec}$, and was followed by a 10 -sec blank interval before the commencement of the next trial. A video recording was made of this display, and a spoken cue was dubbed over the sequence of warning signals for each trial, indicating whether the sequence of letters or the sequence of words would subsequently have to be recalled. This recording was shown to the subjects on monochrome television monitors; the resulting characters were roughly $14 \mathrm{~mm}$ in height, the letters and words were separated by $125 \mathrm{~mm}$, and the monitors were viewed at a distance of $1.5-2.0 \mathrm{~m}$.

For the first part of the experiment, 20 subjects were tested in four equal groups on all 40 trials. Two of these groups viewed the video recording with the sound track, and were told to remember either the sequence of letters or the sequence of words, as appropriate. The other two groups viewed the video recording without the sound track, and were told to remember both the sequence of letters and the sequence of words on each trial, but only to recall the sequence indicated by the subsequent recall signal. All of the subjects were instructed to recall during the subsequent 30 -sec period by writing down the relevant items in the order of presentation and in the positions corresponding to those of presentation on a prepared response sheet. The 24 relevant stimuli were printed at the top of this response sheet as four sets of six items, following the procedure of Baddeley (1968).

Since this first stage of the experiment used a conventional group-testing procedure, the subjects were not individually supervised. It is therefore possible that they did not consistently recall the sequences of items strictly in the order of presentation. To check whether this had influenced their results, the remaining 20 subjects were tested individually, using oral recall. Some of the original subjects also complained of boredom and fatigue toward the end of the testing period, and therefore this second stage of the experiment employed only the first 24 trials. As before, half of the subjects viewed the video recording with the sound track, and half viewed it without the sound track. All of the subjects were instructed to recall the appropriate sequence by saying the items aloud in the order of presentation; the 24 relevant stimuli were displayed as four sets of six items on a sheet of paper that was visible throughout the experiment.

\section{Results}

Table 1 summarizes the performance of the subjects in the two parts of Experiment 1. It is clear that a concurrent memory load produced a substantial overall reduction in performance, and a considerable attenua- 
Table 1

Mean Percentages Correct in Immediate Serial Recall of Phonemically Distinct and Similar Letters and Words, With and Without Concurrent Memory Load (Experiment 1)

\begin{tabular}{|c|c|c|c|c|c|c|}
\hline & \multicolumn{3}{|c|}{ Written Recall } & \multicolumn{3}{|c|}{ Oral Recall } \\
\hline & Distinct & Similar & Difference & Distinct & Similar & Difference \\
\hline \multicolumn{7}{|c|}{ Letters } \\
\hline Memory Load & 29.5 & 30.5 & -1.0 & 30.6 & 27.8 & 2.8 \\
\hline No Load & 88.2 & 65.8 & $22.3 \dagger$ & 83.1 & 64.4 & $18.6^{*}$ \\
\hline \multicolumn{7}{|c|}{ Words } \\
\hline Memory Load & 37.8 & 24.0 & $13.8^{* *}$ & 37.8 & 24.4 & $13.3^{*}$ \\
\hline No Load & 84.7 & 58.2 & $26.5 \dagger$ & 79.2 & 51.7 & $27.5 \dagger$ \\
\hline
\end{tabular}

${ }^{*} p<.05 . \quad{ }^{* *} p<.01 . \quad \dagger_{p}<.001$.

tion of the absolute magnitude of the phonemic similarity effect. Conversely, the effect of a concurrent memory load was more pronounced in the case of phonemically distinct sequences of items than in the case of phonemically confusable sequences of items. There was no material difference between the two parts of the experiment either in terms of the overall level of performance or in terms of the pattern of results obtained. There was thus no evidence that the subjects tested in groups using written recall had failed to recall the sequences strictly in the order of presentation, or that their performance had been reduced by fatigue on later trials. However, there are some interesting differences between the patterns of results obtained with sequences of letters and those obtained with sequences of words, and these differences require elucidation by means of detailed statistical analysis. Since performance in the two parts of the experiment was based upon different (although overlapping) samples of stimulus material, a separate analysis of variance was carried out upon the data collected in each of the two parts. In each case, the design included two random factors, subjects and sequences (the actual sampling fractions for the latter factor being $5 / 720$ and $3 / 720$, respectively). The first random factor was nested under the fixed factor of memory load. The second random factor was nested under the fixed factors of vocabulary (letters or words), confusability of the material to be recalled, and confusability of the material that was presented concurrently with that to be recalled. The dependent variable was the number of items recalled in the correct serial position by each subject on each sequence.

The subjects who were tested in groups with written recall produced significant effects of memory load [quasi $F(1,23)=69.07, p<.001$ ], confusability of the material to be recalled [quasi $F(1,47)=27.63, p<.001$ ], and confusability of the concurrent material $[\mathrm{F}(1,32)=$ $8.97, \mathrm{p}<.01]$. There was also a significant interaction between the first two effects [quasi $F(1,42)=11.08$, $\mathrm{p}<.005$ ], indicating that a concurrent memory load reduced the effect of phonemic similarity, and that the effect of a concurrent memory load was reduced with confusable sequences of items. There was a tendency for sequences of words to show a more pronounced effect of confusability [quasi $F(1,49)=3.24, p<.1$ ], but no other term in the analysis even approached statistical significance. In particular, although performance was rather better when the concurrent material was phonemically confusable $(55.5 \%$ correct) than when it was phonemically distinct $(49.2 \%$ correct $)$, this effect did not interact significantly with that of memory load $[F(1,32)=2.32, p>.1]$. Similarly, the subjects who were tested individually with oral recall produced significant effects of memory load [quasi $F(1,30)=$ $66.03, p<.001]$ and confusability of the material to be recalled $[F(1,16)=24.49, p<.001]$, as well as a significant interaction between these two effects $[F(1,16)$ $=8.04, p<.02]$. No other term in the analysis approached statistical significance.

Directional a posteriori tests (equivalent to onetailed $t$ tests) produced an identical pattern of results for the two parts of the experiment; accordingly, the results of these tests are reported together, and in each case the result obtained with written recall precedes that obtained with oral recall. The effect of memory load was significant for both distinct sequences [quasi $F(1,51)=79.06$, $p<.001$; and quasi $F(1,51)=60.14, p<.001]$ and confusable sequences [quasi $F(1,51)=34.42, p<.001$; and quasi $F(1,51)=27.98, p<.001]$. The effect of confusability of the material to be recalled was significant with no memory load for both sequences of letters [quasi $F(1,93)=16.68, p<.001 ;$ and quasi $F(1,68)=5.45$, $\mathrm{p}<.02$ ] and sequences of words [quasi $F(1,93)=23.42$, $\mathrm{p}<.001 ;$ and quasi $\mathrm{F}(1,68)=11.54, \mathrm{p}<.001]$, and with a memory load for sequences of words [quasi $F(1,93)$ $=6.61, \mathrm{p}<.01$; and quasi $\mathrm{F}(1,68)=2.95, \mathrm{p}<.05]$, but not with a memory load for sequences of letters $(F<1$ in both cases).

\section{Discussion}

This experiment has demonstrated that the effect of phonemic similarity in immediate serial recall is reduced by imposing a second serial-learning task as a concurrent memory load. Conversely, the effect of a concurrent memory load upon performance in immediate serial recall was greater when phonemically distinct sequences 
had to be recalled than when phonemically confusable sequences had to be recalled. These results imply, respectively, that the phonemic store contributing to performance in immediate serial recall is of limited capacity, and that the component of short-term memory that is impaired by a concurrent memory load has access to phonemic coding. On a global view of the results, therefore, Experiment 1 has provided further evidence that phonemic similarity and a concurrent memory load are convergent operational indicators of a limited-capacity phonemic response buffer (cf. Hitch, 1980). The confusability of the concurrent material was positively related to performance on the criterion task for the subjects who were tested in groups using written recall. This occurred even when the concurrent material did not constitute a memory load, which suggests that it might have been phonemically encoded even when it was irrelevant to the task in hand. However, no effect of the confusability of the concurrent material was obtained in Experiment 1 when the subjects were tested individually using oral recall, and no such effect was obtained in Experiment 2, reported below.

The detailed findings of Experiment 1 are somewhat more complicated, and separate attention must be given to the results obtained with sequences of letters and with sequences of words. In the immediate recall of sequences of letters, a memory load consisting of a sequence of words drastically reduced the overall level of performance and completely removed the phonemic similarity effect. This suggests that the subjects operating under a memory load tended to devote the capacity of the phonemic response buffer exclusively to the storage of the concurrent sequences of words. Moreover, the substantial impairment observed even in the case of phonemically confusable sequences (which presumably could not be efficiently represented in a phonemic store) indicates that the concurrent sequences of words also attenuated the storage capacity of the central executive processor. The residual retention of roughly two items from each sequence of letters might be attributed to the employment of a restricted visual code of the sort discussed by Hiles (1974), by Posner, Boies, Eichelman, and Taylor (1969), and by Warrington and Shallice (1972). Consistent with this, Vallar and Baddeley (1982) found that performance in the Peterson task with three visually presented consonants was disrupted by counting backward but not by articulatory suppression. They concluded that the maintenance of such a limited visual sequence was achieved by the central executive processor without the support of the phonemic response buffer.

However, since the subjects who operated under a concurrent memory load in the present study do not appear to have been using phonemic coding to remember the sequences of letters, their impaired performance on the sequences of words cannot be ascribed to any reduction in the availability of storage space within the phonemic response buffer. Indeed, in the immediate serial recall of sequences of words, a memory load consisting of a sequence of letters did not remove the phonemic similarity effect. Nevertheless, such a memory load significantly reduced the absolute magnitude of that effect, as well as disrupting the overall level of performance. This suggests that the concurrent task reduced the capacity of the central executive processor to encode material into the phonemic response buffer. In other words, since the concurrent sequences of letters showed no sign of any phonemic similarity effect when they were presented as a memory load, they do not seem to have gained access to the phonemic response buffer, but instead appear to have disrupted the efficient phonological encoding of the sequences of words.

In short, a concurrent memory load may have any one of three different effects upon the working-memory system. First, like any other concurrent task, it may occupy some of the capacity of the central executive processor. Second, like concurrent articulatory suppression, it may disrupt the translation of orthographic stimuli into a phonological representation. Third, since the concurrent material may itself be translated into such a representation, it may compete for storage capacity within the phonemic response buffer.

\section{EXPERIMENT 2}

The alternative basis for characterizing the phonemic response buffer is its organizational properties. It is well known that the effects of phonemic coding are most clearly observed in situations that require the retention of serial-order information, and it is thus not surprising that Baddeley and Hitch (1974) assumed that the mechanism postulated to explain those effects should represent different stimulus items in their order of presentation. However, whether the phonemic response buffer contributes to performance in tasks that do not require the retention of serial-order information is then open to question.

From a purely logical point of view, the retention of serial-order information is sufficient (although not, of course, necessary) for the accurate recall of item information. One might therefore expect that a mechanism with the characteristics attributed to the phonemic response buffer would contribute to performance even in the case of tasks that did not explicitly demand the retrieval of serial-order information. A more extreme point of view is contained in the "perturbation" theory of Estes (1972; Lee \& Estes, 1981), which holds that "loss of item information is a byproduct of the loss of positional or order information." On this account, all representations in short-term memory are essentially organized on a sequential basis. Within the program of research described by Baddeley and Hitch (1974), it was observed that performance in free recall was impaired by a concurrent memory load and by articulatory suppression, but was improved by phonemic similarity. These results were taken to imply that the phonemic response buffer was involved in free recall, 
although Baddeley and Hitch demonstrated that it was not responsible for the recency effect.

Nevertheless, it must be acknowledged that the effects of phonemic similarity, a concurrent memory load, articulatory suppression, and word length tend to be much less pronounced in free recall than in serial recall (see Baddeley \& Hitch, 1974, 1977; Baddeley et al., 1975; Craik, 1968; Craik \& Levy, 1970; Richardson \& Baddeley, 1975; Richardson et al., 1980). Indeed, the positive effect of phonemic similarity in free recall is normally attributed to its use as an organizational principle in long-term memory. Moreover, the rather tenuous effects of the other three variables in free recall can be ascribed to the central executive processor rather than to the phonemic response buffer. For example, Baddeley and Hitch (1974) found that a concurrent memory load of one, two, or three items produced very little impairment in reasoning, comprehension, or free recall. To explain this, they proposed that the maintenance of stimulus items from the concurrent serial-recall task was carried out in the phonemic response buffer, thus releasing the central executive processor for the performance of the criterion task. Accordingly, impaired performance would be obtained in a criterion task demanding central processing capacity only when a concurrent memory load exceeded the capacity of the phonemic response buffer and required the support of general-purpose work space within the central executive processor. In particular, the maintenance of up to three items within the phonemic response buffer was assumed to make relatively few demands upon the capacity of the central executive processor (Baddeley, 1976, p. 177; Hitch, 1980; Hitch \& Baddeley, 1977, p. 98; cf. Baddeley, 1981).

On this account, it would appear that the storage capacity of the phonemic response buffer is available for the maintenance of a concurrent load of up to three stimulus items in tests of reasoning, comprehension, and free recall, with no appreciable reduction in the level of performance. This line of argument seems to imply that the phonemic response buffer does not itself make any material contribution to performance in those criterion tasks after all. On the contrary, the phonemic response buffer might contribute to performance in a significant manner only in criterion tasks that explicitly require the retention of serial-order information (see Baddeley \& Lewis, 1981). Evidence on this point was collected by Klapp et al. (1983), who used the "missing scan" procedure devised by Buschke (1963). In this task, the subject is presented with eight of the nine nonzero decimal digits in a random order, and is asked to determine which digit was omitted. Klapp et al. found that performance on this task was not affected by rhythmic grouping of the stimulus items or by articulatory suppression. This suggested that, although the recall of a sequence of items in the order of presentation might be mediated by the sort of phonemic response buffer described by Baddeley and Hitch (1974), the recall of a sequence of items in any order (such as would be required to identify the missing digit) was not mediated by such a store. On the contrary, they argued, tasks that do not require the retention of serial-order information depend exclusively upon the residual component or components of working memory.

In general, it can be assumed that, if the phonemic response buffer is involved in a particular task, then that task should disrupt the contribution of the phonemic response buffer to performance in a second, concurrent task in which it is also involved. This methodological approach underlay Baddeley and Hitch's (1974) choice of immediate serial recall as a concurrent task. However, if the phonemic response buffer is involved in free recall, then that task too should disrupt the contribution of the phonemic response buffer to performance in a criterion task. In particular, a concurrent free-recall task should attenuate the effects of phonemic coding in immediate serial recall. On the alternative hypothesis, that the phonemic response buffer is not involved in free recall, this task should not disrupt the contribution of the phonemic response buffer to performance in a criterion task. In particular, a concurrent free-recall task should not attenuate the effects of phonemic coding in immediate serial recall. There might be a general reduction in performance on the latter task, since storage capacity within the central executive processor might no longer be available to support the capacity of the phonemic response buffer (see Baddeley, 1976, p. 179). Moreover, the subjects' overt or covert rehearsal of items from the concurrent free-recall task might constitute a form of articulatory suppression and prohibit the encoding of visually presented items in the form of phonological representations within the phonemic response buffer (Baddeley, Note 1). Nevertheless, a concurrent free-recall task should not attenuate the effects of phonemic coding in the immediate serial recall of auditorily presented sequences.

Experiments in which subjects were required to carry out a free-recall task and a serial-recall task simultaneously were reported by Baddeley and Hitch (1974, 1977), but these papers do not contain any data relevant to the above predictions. First, the experiments were designed to investigate the effects of a concurrent memory load upon free recall, and the performance of subjects on the concurrent serial-recall task was not reported for any of the experiments. Second, Baddeley and Hitch required their subjects to maintain "high accuracy" on the concurrent serial-recall task; it is therefore likely that performance on this task was at a ceiling, and the results would have been relatively uninformative concerning the mechanisms and processes being employed. Finally, none of these experiments included an independent procedure for evaluating the contribution of the phonemic response buffer to either of the tasks involved. To satisfy the latter requirement, Experiment 2 manipulated the phonemic confusability of sequences of letters presented in the auditory and 
visual modalities, to be learned with and without the concurrent free recall of lists of unrelated words. As in the original studies by Baddeley and Hitch (1974, 1977), the serial-recall task was expected to impair the subjects' performance in immediate free recall, but the crucial question was whether the concurrent free-recall task would attenuate the phonemic similarity effect in the immediate serial recall of auditorily presented sequences.

Although the effects of phonemic similarity, a concurrent memory load, articulatory suppression, and word length in free recall are somewhat tenuous, little is known about the effects of irrelevant, unattended speech. In the immediate serial recall of visually presented material, such a manipulation produces a substantial impairment, but only on phonemically distinct sequences (Colle \& Welsh, 1976), and not under conditions of articulatory suppression (Salamé \& Baddeley, 1982). These results suggest that irrelevant, unattended speech disrupts the contribution of the phonemic response buffer to performance in immediate serial recall. The following experiment considered the possibility of a similar effect in immediate free recall by incorporating two control conditions that differed with respect to the presence or absence of irrelevant stimuli. If the phonemic response buffer is involved in immediate free recall, then there should be a clear impairment on this task specifically associated with the occurrence of unattended speech. On the other hand, if unattended speech has no such effect upon performance, or if irrelevant visual stimuli produce a similar impairment, then there will be no good evidence for a selective involvement of the phonemic response buffer in immediate free recall.

\section{Method}

Design. The subjects were presented simultaneously with sequences of letters for immediate serial recall and lists of words for immediate free recall. The phonological confusability of the letters and the type of material to be recalled were orthogonally varied in an unpredictable fashion across different trials following a within-subjects design. The sequences of letters were presented visually to half of the subjects and auditorily to the remainder; the lists of words were always presented visually. Independently of this, one-third of the subjects were presented only with the material to be recalled and were not exposed to the irrelevant stimuli. One-third of the subjects were presented with sequences of letters and lists of words simultaneously, but the material to be recalled on each trial was identified in advance; thus, there was no concurrent memory task, but the subjects were exposed to irrelevant stimuli. Finally, one-third of the subjects were presented with sequences of letters and lists of words simultaneously, but the material to be recalled on each trial was not identified in advance, and thus the subjects were operating with a concurrent memory task.

Subjects. This experiment involved 60 students applying for admission to the Psychology Honours Degree course at Brunel University who volunteered to take part.

Materials. Ten random orderings were produced from each of the two sets of letters employed in Experiment 1. In addition, 240 English nouns rated $\mathrm{AA}$ or $\mathrm{A}$ in frequency according to the norms of Thorndike and Lorge (1944) were assigned at random to 20 lists, each of 12 words. The sequences of letters and the lists of words were assigned in pairs at random to 20 trials, with the constraint that 2 of every successive set of 4 trials involved each of the sets of letters; 1 of these trials was randomly assigned for letter recall, and the other for word recall.

Procedure. The subjects were given standard serial-recall instructions for the sequences of letters and standard free-recall instructions for the lists or words. They were tested in groups of two or three, and they were allowed $40 \mathrm{sec}$ on each trial to write down their responses on a prepared sheet, at the top of which were printed the two sets of six letters.

For the first part of this experiment, the sequences of letters and the lists of words were presented visually. The stimuli were prepared and presented as in Experiment 1, except that the words were presented at a rate of $1 \mathrm{item} / \mathrm{sec}$ and that a letter appeared above every alternate word, beginning with the first. As before, a spoken cue was dubbed over the sequence of visual warning signals for each trial, indicating whether the sequence of letters or the list of words would subsequently have to be recalled. Ten subjects viewed the video recording without the sound track and were told to remember both the sequence of letters and the list of words on each trial, but only to recall the material indicated by the subsequent visual recall signal. Another 10 subjects viewed the video recording with the sound track and were told to remember either the sequence of letters or the list of words, as appropriate. Finally, another 10 subjects received the same instructions as the second group, but a mask was used to cover the area of the television screen where the irrelevant material would appear on each trial. Thus, the first and second groups were exposed to both the sequence of letters and the list of words on each trial, but the third group was not.

For the second part of the experiment, the sequences of letters were presented auditorily, but the lists of words were presented visually. The sequences of letters were recorded onto the sound track of the videotape in a male voice such that they coincided with their visual presentation (that is, at a rate of $2 \mathrm{sec} / \mathrm{item})$, and the area of the television screen where they would have appeared was permanently masked. The sound track also contained the spoken cue dubbed over the sequence of visual warning signals for each trial, indicating whether the sequence of letters or the list of words would subsequently have to be recalled. A new group of 10 subjects viewed the video recording with the sound track switched off during the sequence of warning signals for each trial and were told to remember both the sequence of letters and the list of words, but only to recall the material indicated by the subsequent visual recall signal. Another 10 subjects viewed the video recording with the complete sound track and were told to remember either the sequence of letters or the list of words on each trial, as appropriate. Finally, another 10 subjects received the same instructions as the second group, but the irrelevant material was removed by masking the appropriate area of the television screen or by switching off the sound track of the video recording. Thus, once again, the first and second groups were exposed to both the sequence of letters and the list of words on each trial, but the third group was not.

\section{Results and Discussion}

Serial recall. Table 2 summarizes the performance on the sequences of letters presented for immediate serial recall. When the sequences were presented visually, the concurrent free recall of lists of unrelated words produced a large reduction in performance, and it completely eliminated the phonemic similarity effect. However, when the sequences were presented auditorily, the concurrent free recall of lists of unrelated words produced a similar reduction in overall performance without abolishing the phonemic similarity effect. To confirm the reliability of these findings, an analysis of variance was carried out upon the number of items 
Table 2

Mean Percentages Correct in Immediate Serial Recall of Phonemically Distinct and Similar, Visual and Auditory Sequences of Letters, With and Without Concurrent Free Recall of Visually Presented Lists of Words (Experiment 2)

\begin{tabular}{lccc}
\hline & Distinct & Similar & Difference \\
No Irrelevant Stimuli & Visual Sequences & & $12.3^{*}$ \\
Irrelevant Stimuli & 88.0 & 75.7 & $11.7^{*}$ \\
Concurrent Free Recall & 84.7 & 73.0 & -3.0 \\
& 40.3 & 43.3 & $15.3^{* *}$ \\
No Irrelevant Stimuli & Auditory Sequences & 77.3 & $15.7^{* *}$ \\
Irrelevant Stimuli & 92.7 & 76.7 & $12.0^{*}$ \\
Concurrent Free Recall & 92.3 & 42.0 & 54.0 \\
\hline
\end{tabular}

${ }^{*} p<.05 . \quad{ }^{* *} p<.01$.

recalled in the correct serial position by each subject on each sequence of letters. The experimental design included two random factors, subjects and sequences (the actual sampling fraction for the latter factor being $5 / 720$ ). The first random factor was nested under the fixed factors of presentation groups and stimulus modality, and the second random factor was nested under the fixed factor of phonemic confusability.

Directional tests demonstrated significant effects of stimulus modality $[F(1,54)=3.14, p<.05]$ and of phonemic confusability [quasi $\mathrm{F}(1,20)=9.52, \mathrm{p}<.005$ ] however, the interaction between these two effects approached statistical significance $[\mathrm{F}(1,54)=2.89$, $p<.1$ ], indicating that stimulus modality affected performance only in the case of phonemically distinct sequences of letters. There was also significant variation among the three presentation groups [quasi $F(2,62)=$ $49.27, p<.001]$. A posteriori tests found no sign of any difference between the two control groups in terms of either the main effect of irrelevant stimuli or its interaction with the remaining independent variables $(F<1$ in every case). The results of these two groups were therefore combined in evaluating the effect of the concurrent free recall of lists of unrelated words, which proved to be highly significant [quasi $F(1,62)=98.27$, $\mathrm{p}<.001]$. Directional tests showed that the interaction between the effect of the concurrent free recall of lists of unrelated words and that of phonemic confusability was significant for visual sequences $[F(1,54)=$ $5.37, \mathrm{p}<.02]$, but not for auditory sequences $(\mathrm{F}<1)$. With a concurrent free-recall task, the effect of phonemic confusability was significant under auditory presentation [quasi $F(1,102)=2.89, \mathrm{p}<.05$ ], but not under visual presentation $(F<1)$. With no concurrent free-recall task, the effect of phonemic confusability was significant under both auditory presentation [quasi $\mathrm{F}(1,102)=8.87, \mathrm{p}<.005]$ and visual presentation [quasi $F(1,102)=5.45, p<.02$ ]

This experiment demonstrated that performance in the immediate serial recall of sequences of letters is severely disrupted by the concurrent free recall of lists of unrelated words. This impairment was observed even in the case of phonemically confusable sequences of letters, which suggests that a primary locus of the effect is the storage capacity of the central executive processor. In the case of auditorily presented sequences of letters, there was no significant reduction in either the absolute or the relative magnitude of the phonemic similarity effect, which implies that the concurrent free recall of lists of unrelated words does not compete for the storage capacity of the phonemic response buffer. This, in turn, entails that the phonemic response buffer is not involved in immediate free recall. In the case of visually presented sequences of letters, the phonemic similarity effect was completely eliminated, which suggests that the concurrent free recall of lists of unrelated words prohibits the translation of orthographic stimuli into a phonological representation. A plausible explanation for this is that the overt or covert rehearsal of the lists of words presented for immediate free recall constituted a form of articulatory suppression. Finally, it may be noted that there was no sign of any effect of irrelevant visual stimuli upon the immediate serial recall of either auditory or visual sequences of letters.

Free recall. Table 3 summarizes the performance on the lists of unrelated words presented for immediate free recall, averaged across the primacy, middle, and recency portions of the serial-position function. It is clear that a concurrent memory load of six letters produced a substantial reduction in performance throughout the lists of words. The presence of irrelevant stimuli that did not constitute a memory load produced a much less pronounced decrement that was more apparent with irrelevant visual stimuli than with irrelevant speech.

These effects were evaluated by means of an analysis of variance upon each subject's performance on each word. The experimental design included two random factors, subjects and lists, and one fixed factor, serial positions. The first random factor was nested under the fixed factors of presentation groups and modality of the concurrent material. The second random factor was nested under the fixed factor of confusability of the concurrent material. There was a significant effect of serial positions [quasi $F(11,157)=15.42, \mathrm{p}<.001$ ], and significant variation among the three presentation groups $[F(2,54)=80.69, p<.001]$. The latter effect 
Table 3

Mean Percentages Correct in Immediate Free Recall of Visually Presented Lists of Words With and Without Concurrent Memory Load of Visual and Auditory Sequences of Letters (Experiment 2)

\begin{tabular}{|c|c|c|c|c|}
\hline & \multicolumn{3}{|c|}{ Serial Positions } & \multirow[b]{2}{*}{ Overall } \\
\hline & 14 & $5-8$ & $9-12$ & \\
\hline \multicolumn{5}{|c|}{ Concurrent Visual Sequences } \\
\hline $\begin{array}{l}\text { No Irrelevant Stimuli } \\
\text { Irrelevant Stimuli } \\
\text { Concurrent Memory Load }\end{array}$ & $\begin{array}{l}57.3 \\
46.3 \\
21.0\end{array}$ & $\begin{array}{l}33.5 \\
24.8 \\
12.5\end{array}$ & $\begin{array}{l}62.3 \\
55.5 \\
35.3\end{array}$ & $\begin{array}{l}51.0 \\
42.2 \\
22.9\end{array}$ \\
\hline \multicolumn{5}{|c|}{ Concurrent Auditory Sequences } \\
\hline $\begin{array}{l}\text { No Irrelevant Stimuli } \\
\text { Irrelevant Stimuli } \\
\text { Concurrent Memory Load }\end{array}$ & $\begin{array}{l}55.8 \\
42.8 \\
33.0\end{array}$ & $\begin{array}{l}27.8 \\
29.3 \\
20.3\end{array}$ & $\begin{array}{l}50.3 \\
57.8 \\
32.0\end{array}$ & $\begin{array}{l}44.6 \\
43.3 \\
28.4\end{array}$ \\
\hline
\end{tabular}

interacted with that of serial positions [quasi $F(36,610)$ $=1.69, \mathrm{p}<.01]$ and with that of modality of the concurrent material $[\mathrm{F}(2,54)=5.46, \mathrm{p}<.01]$, and there was also a three-way interaction involving all three of these factors $[F(22,594)=1.64, p<.05]$.

A posteriori comparisons between the two control groups produced a significant main effect of irrelevant stimuli $[F(1,54)=7.77, p<.01]$, which interacted with the effect of modality of the concurrent material $[\mathrm{F}(1,54)$ $=4.23, \mathrm{p}<.05]$, but not with that of serial positions [quasi $F(24,610)=1.13, \mathrm{p}>.25$ ]. The simple main effect of irrelevant stimuli was significant with irrelevant visual stimuli $[\mathrm{F}(1,54)=11.71, \mathrm{p}<.005]$, but not with irrelevant auditory stimuli $(\mathrm{F}<1)$. Table 3 shows that the occurrence of irrelevant visual stimuli produced a reduction in performance throughout the lists of words, but that the occurrence of irrelevant auditory stimuli produced no such impairment. Conversely, the simple main effect of modality of the concurrent material was significant with no irrelevant stimuli [quasi $F(1,78)=$ $4.62, \mathrm{p}<.05]$, but not with irrelevant stimuli $(\mathrm{F}<1)$. Table 3 shows that, with no irrelevant stimuli, the sub. jects were apparently impaired by having to switch from the auditory modality to the visual modality in order to carry out the free-recall task.

When the subjects were exposed to irrelevant auditory stimuli, the overall level of performance was $45.3 \%$ correct at the odd-numbered serial positions and $41.3 \%$ correct at the even-numbered serial positions. The corresponding levels of performance with no irrelevant auditory stimuli were $44.0 \%$ correct and $45.2 \%$ correct, respectively. Thus, the occurrence of irrelevant auditory stimuli produced no decrement in performance either on the words with which those stimuli were synchronized or on the words with which they alternated (cf. Salamé \& Baddeley, 1982, Experiments 1 and 2). The failure of unattended speech to reduce performance in the immediate free recall of lists of unrelated words suggests that the phonemic response buffer is not involved in this task. In this experiment, irrelevant stimuli impaired performance in immediate free recall only when they were presented in the same (visual) modality as the lists of words to be learned, which suggests that their effect upon cognitive processing was limited to competition for modality-specific attentional capacity (Martin, 1980; Navon \& Gopher, 1979; Treisman \& Davies, 1973)

A posteriori comparisons between the two groups who were exposed to irrelevant stimuli produced a significant main effect of a concurrent memory load $[F(1,54)=87.28, p<.001]$, which interacted with the effect of serial positions [quasi $F(18,610)=1.71$, $p<.05]$, but not with that of modality of the concurrent material $[F(1,54)=1.47, p>.2]$. Nevertheless, the effect of a concurrent memory load was significant for the early serial positions (14) [quasi $F(1,685)=$ $24.71, p<.001]$, for the middle serial positions $(5-8)$ [quasi $F(1,685)=9.31, p<.005$ ], and for the final serial positions $(9-12)$ [quasi $F(1,685)=42.29, p<.001]$. Table 3 shows that this effect was somewhat greater at the early and final serial positions, although this was probably the result of a floor effect at the middle serial positions; indeed, the proportional decrement in performance was roughly constant throughout the lists of words. Thus, the present experiment has replicated the finding of Baddeley and Hitch $(1974,1977)$ that a concurrent memory load impairs performance in immediate free recall without abolishing the recency effect.

Nevertheless, this experiment has provided two convergent sorts of evidence against the idea that the phonemic response buffer that was postulated by Baddeley and Hitch (1974) is involved in immediate free recall. First, a concurrent free-recall task did not attenuate the phonemic similarity effect obtained in the immediate serial recall of auditorily presented sequences of letters. Second, irrelevant, unattended speech had no effect at all upon performance in the immediate free recall of visually presented lists of unrelated words. These findings indicate that the recall of item information may be achieved without the support of a sequentially organized buffer store. This contradicts the assumption of the "perturbation" theory of Estes (1972; Lee \& Estes, 1981) that representations in short- 
term memory are essentially organized on the basis of temporal relationships. More generally, the present findings are entirely in accordance with the conclusion of Klapp et al. (1983) that tasks that do not formally require the retention of serial-order information are not mediated by the phonemic response buffer, but depend exclusively upon the residual components of working memory.

\section{GENERAL CONCLUSIONS}

Phonemic similarity, articulatory suppression, a concurrent memory load, word length, and unattended speech individually impair performance in a variety of tasks. They also interact in a meaningful fashion in terms of their effects in immediate serial recall. The model of working memory that was proposed by Baddeley and his colleagues provides an elegant and parsimonious explanation of these various empirical phenomena in terms of two hypothetical mechanisms, a central executive processor and a phonemic response buffer. The importance of any program of empirical research on the role of working memory in human information processing is therefore to be evaluated in terms of the extent to which it enhances our understanding of the nature of these two components and their interaction with each other.

The original concept of the phonemic response buffer was that of a subsidiary slave system that was able to maintain verbal material by means of subvocal rehearsal (Baddeley, 1981). Although it was proposed that the form of coding characteristic of this component was related to the mechanisms underlying speech production, the effects of articulatory suppression and of unattended speech implicate a more abstract phonemic representation that is accessible either through an obligatory encoding of auditory stimuli or through an optional process of subvocal articulation (Baddeley et al., 1975; Richardson et al., 1980; Salamé \& Baddeley, 1982). The form of organization characteristic of this component was assumed to be temporal and serial. This notion is supported by the finding of Klapp et al. (1983) that articulatory suppression did not affect performance in the "missing scan" task. It was confirmed by the results of Experiment 2, which found no effect of unattended speech upon the overall level of performance in free recall, and no effect of a concurrent free-recall task upon the magnitude of the phonemic similarity effect in the serial recall of auditory sequences. The effects of a concurrent memory load upon performance in tests of reasoning, comprehension, and free recall (Baddeley \& Hitch, 1974, 1977) suggest that the storage capacity of the phonemic response buffer is between three and six items. However, this parameter is likely to vary among the normal population, and it is thus not surprising that some subjects seem to be able to hold a memory load of seven or eight items with no apparent reduction in verbal-reasoning performance (Baddeley, 1981).

The arguments and evidence presented in this paper suggest that the function of the phonemic response buffer is merely that of supplementing the storage capacity of the central executive processor in a somewhat restricted range of cognitive tasks. One might therefore conclude that the phonemic response buffer is a relatively peripheral and unimportant component of the total working-memory system that is employed only when the central processing capacity of the subject is overloaded. Similar assessments of the role of shortterm phonemic storage in human information processing have been given by Craik (1973) and by Weiskrantz (1970). In general, it would appear that the phonemic response buffer makes a significant contribution to performance only in cognitive tasks that depend upon the accurate retention of order information. This explains why subjects can retain a memory load of up to three items with no apparent reduction in their performance in tests of reasoning, comprehension, and free recall, and why phonemic similarity, word length, and unattended speech fail to disrupt performance in free recall (Baddeley \& Hitch, 1974, 1977; Craik, 1968; Craik \& Levy, 1970). The positive effect of phonemic similarity in free recall is attributable to its role as an organizational principle in long-term storage. The reliable, although far from devastating, effects of larger memory loads and of articulatory suppression may be ascribed to their disruption of the storage capacity of the central executive processor; a similar disruption is seen in the effects of articulatory suppression upon the immediate serial recall of auditory sequences of items (Baddeley et al., 1975; Richardson et al., 1980).

In contrast, the operating characteristics of the central executive processor have been much less clearly defined and have thus proved to be more difficult to investigate experimentally. Levy (1978) observed that functions have been ascribed to the central executive processor without any detailed specification of how they might be performed, and Baddeley (1981) acknowledged that this component represents "the area of our residual ignorance" about working memory. Indeed, he elegantly defined the task facing future research aimed at reducing this area of ignorance: "An adequate theory of the Central Executive would probably include not only a specification of its method of manipulating control processes and integrating the growing number of peripheral systems, but would also require an understanding of selective attention and probably of the role and function of consciousness." One of the reasons for equivocation over the operating characteristics of the central executive processor is that its functions are apparently highly task specific. Thus, Hitch (1978) was able to give a very detailed account of the operations to be attributed to the central executive processor when subjects are required to carry out mental arithmetic. As another 
example, the central executive processor appears to employ rhythmic grouping of the stimulus items in immediate serial recall, but not in the "missing scan" procedure investigated by Klapp et al. (1983). To a very large extent, therefore, the future development of this concept will depend upon the ingenuity of theorists in identifying formal similarities among different experimental tasks, and in defining general-purpose heuristic procedures that might underlie the task-dependent properties of human cognitive processing.

At present, one can say that the central executive processor is the more important of the two components of working memory originally postulated by Baddeley and Hitch (1974), and that is has a crucial role in decision making, problem solving, and long-term storage. That it has direct access to prelexical phonology in reading individual items is suggested by the ability of normal subjects to carry out rhyme judgments involving nonwords even under conditions of articulatory suppression; that it has direct access to postlexical phonology is suggested by the ability of normal subjects to carry out similar judgments involving words with irregular spellings (Baddeley \& Lewis, 1981; Coltheart, 1980). Nevertheless, the central executive processor does not appear to have direct access to phonological information as a means of encoding verbal material in short-term memory (Baddeley et al., 1975). With auditory presentation, such information is available to the phonemic response buffer in a direct and obligatory fashion (Salamé \& Baddeley, 1982), which suggests a crucial, although as yet uninvestigated, role for the phonemic response buffer in speech perception. With visual presentation, the availability of phonological information depends upon the use of appropriate control processes that apparently involve subvocal articulation (Baddeley, 1979). In the free recall of visually presented stimuli, the results of Experiment 2 suggest that the central executive processor has direct access to the more abstract encoding operations required for long-term storage without the use of an intermediate phonological code. Finally, the central executive processor appears to have direct access to a restricted visual code that is able to hold up to three items even under conditions of articulatory suppression (Vallar \& Baddeley, 1982).

An important assumption in the concept of the central executive processor is that it represents a pool of general processing capacity to be shared among the various tasks confronting an individual subject. This implies that two concurrent tasks should manifest a direct tradeoff in the form of a negative correlation between the levels of performance achieved (cf. Navon \& Gopher, 1979; Norman \& Bobrow, 1975). Baddeley (1981) mentioned the results of an unpublished experiment that suggested a positive correlation across different subjects between verbal reasoning and immediate serial recall. However, if the former task utilized the central executive processor, whereas the latter depended largely upon the phonemic response buffer, this finding merely represents a direct relationship between the capacities of the two components. A more crucial test of a tradeoff in the capacity of the central executive processor is to be found in the results of Experiment 2 of the present investigation. The 10 subjects who carried out the immediate free recall of lists of words at the same time as they attempted to retain visually presented sequences of letters were apparently not using the phonemic response buffer for either task. A comparison of their overall performance on the two concurrent tasks produced a significant negative correlation of -.64 $[F(1,8)=5.66, p<.05]$, which is consistent with the hypothesis of a pool of general processing capacity. Nevertheless, the computation of a correlation across different experimental subjects is only a valid test of this hypothesis if the size of the pool is constant. Otherwise, it confounds individual differences in the allocation of the pool to the two concurrent tasks with individual differences in its total size. A less conservative test of the hypothesis would require the computation of correlations within individual subjects across different experimental trials using comparable stimulus materials and constant presentation conditions. In other words, future empirical research on working memory should include a formal psychometric analysis of individual subjects in terms of their deployment of the total capacity of the central executive processor.

\section{REFERENCE NOTE}

\section{Baddeley, A. D. Personal communication, July 1981.}

\section{REFERENCES}

BADDELEY, A. D. How does acoustic similarity influence shortterm memory? Quarterly Journal of Experimental Psychology, $1968,20,249-264$.

BADDELEY, A. D. The psychology of memory. New York: Basic Books, 1976.

BADDELEY, A. D. Working memory and reading. in P. A. Kolers, M. E. Wrolstad, \& H. Bouma (Eds.), Processing of visible language (Vol. 1). New York: Plenum Press, 1979.

BADDELEY, A. D. The concept of working memory: A view of its current state and probable future development. Cognition, 1981, 10, 17-23.

Badpeley, A. D., Eldidiat, M., \& Lewis, V. The role of subvocalization in reading. Quarterly Journal of Experimental Psychology, 1981, 33A, 439-454.

BADDELEY, A. D., \& HrTCh, G. J. Working memory. In G. H. Bower (Ed.), The psychology of learning and motivation: Advances in research and theory (Vol. 8). New York: Academic Press, 1974.

Baddeley, A. D., \& Hitch, G. J. Recency re-examined. In S. Dornic (Ed.), Attention and performance VI. London: Academic Press, 1977.

BADDELEY, A. D., \& LEWIS, V. Inner active processes in reading: The inner voice, the inner ear, and the inner eye. In $\mathbf{A} . \mathbf{M}$. Lesgold \& C. A. Perfetti (Eds.), Interactive processes in reading. Hillsdale, N.J: Erlbaum, 1981.

Baddeley, A. D., Thomson, N., \& Buchanan, M. Word length and the structure of short-term memory. Journal of Verbal Learning and Verbal Behavior, 1975, 14, 575-589.

Besner, D. Basic processes in reading: Two phonological codes. Canadian Journal of Psychology, 1982, 36, 701-711. 
Besner, D., Davies, J., \& Daniels, S. Reading for meaning: The effects of concurrent articulation. Quarterly Journal of Experimental Psychology, 1981, 33A, 415-437.

BUSCHKE, $H$. Relative retention in immediate memory determined by the missing scan method. Nature, 1963, 212, 1129-1130.

Colle, H. A., \& Welsh, A. Acoustic masking in primary memory. Journal of Verbal Learning and Verbal Behavior, 1976, 15, 17-32.

Colthe arT, M. Reading, phonological recoding, and deep dyslexia. In M. Coltheart, K. E. Patterson, \& J. C. Marshall (Eds.), Deep dyslexia. London: Routledge \& Kegan Paul, 1980.

Craik, F. I. M. Two components in free recall. Journal of Verbal Learning and Verbal Behavior, 1968, 7, 996-1004.

Craik, F. I. M. A "levels of analysis" view of memory. In P. Pliner, L. Krames, \& T. Alloway (Eds.), Communication and affect: Language and thought. New York: Academic Press, 1973.

Craik, F. I. M., \& Levy, B. A. Semantic and acoustic information in primary memory. Journal of Experimental Psychology, $1970,86,77-82$.

Eluis, N. C., \& Hennelly, R. A. A bilingual word length effect: Implications for intelligence testing and the relative ease of mental calculation in English and Welsh. British Journal of Psychology, 1980, 71, 43-52.

ESTES, W. K. An associative basis for coding and organization in memory. In A. W. Melton \& E. Martin (Eds.), Coding processes in human memory. Washington, D.C: Winston, 1972.

Hiles, D. R. Visual encoding in short-term memory (Doctoral dissertation, McGill University, 1973). Dissertation Abstracts International, 1974, 34, 6241B.

Hiтch, G. J. The role of short-term working memory in mental arithmetic. Cognitive Psychology, 1978, 10, 302-323.

Hiтch, G. J. Developing the concept of working memory. In G. Claxton (Ed.), New directions in cognitive psychology. London: Routledge \& Kegan Paul, 1980.

Hitch, G. J., \& Baddeley, A. D. Working memory. In Cognitive psychology-A third level course. Milton Keynes, England: Open University Press, 1977.

Klapp, S. T., Marshburn, E. A., \& Lester, P. T. Short-term memory does not involve the "working memory" of information processing: The demise of a common assumption. Journal of Experimental Psychology: General, 1983, 112, 240-264.

LEE, C. L., \& Estes, W. K. Item and order information in shortterm memory: Evidence for multilevel perturbation processes. Journal of Experimental Psychology: Human Learning and Memory, 1981, 7, 149-169.

LEvy, B. A. Role of articulation in auditory and visual shortterm memory. Journal of Verbal Learning and Verbal Behavior, $1971,10,123-132$.

LEVy, B. A. Speech analysis during sentence processing: Reading versus listening. Visible L anguage, 1978, 12, 81-101.
Martin, M. Attention to words in different modalities: Fourchannel presentation with physical and semantic selection. Acta Psychologica, 1980, 44, 99-115.

Murray, D. J. Articulation and acoustic confusability in shortterm meinory. Journal of Experimental Psychology, 1968, 78, 679-684.

Navon, D., \& Gopher, D. On the economy of the human processing system. Psychological Review, 1979, 86, 214-255.

Nicholson, R. The relationship between memory span and encoding speed. In M. Friedman, J. P. Das, \& N. O'Connor (Eds.), Intelligence and learning. New York: Plenum Press, 1981.

Norman, D. A., \& Bobrow, D. G. On data-limited and resourcelimited processes. Cognitive Psychology, 1975, 7, 44-64.

Peterson, L. R., \& Johnson, S. F. Some effects of minimizing articulation on short-term retention. Journal of Verbal Learning and Verbal Behavior, 1971, 10, 346-354.

Posner, M. I., Boies, S. J., Eichelman, W. H., \& Taylor, R. L. Retention of visual and name codes of single letters. Journal of Experimental Psychology Monograph, 1969, 79(1, Pt. 2).

Richardson, J. T. E., \& BADdeley, A. D. The effects of articulatory suppression in free recall. Journal of Verbal Learning and Verbal Behavior, 1975, 14, 623-629.

Richardson, J. T. E., Greaves, D. E., \& Smith, M. M. C. Does articulatory suppression eliminate the phonemic similarity effect in short-term recall? Bulletin of the Psychonomic Society, 1980, 16, 417-420.

Salamé, P., \& Baddeley, A. D. Disruption of short-term memory by unattended speech: Implications for the structure of working memory. Journal of Verbal Learning and Verbal Behavior, 1982, 21, 150-164.

Thonndike, E. L., \& Lorge, I. The teacher's word book of 30,000 words. New York: Teachers College, 1944.

Theisman, A. M., \& Davies, A. Divided attention to ear and eye. In S. Kornblum (Ed.), Attention and performance IV. London: Academic Press, 1973.

Vallar, G., \& Baddeley, A. D. Short-term forgetting and the articulatory loop. Quarterly Journal of Experimental Psychology, 1982, 34A, 53-60.

Warrington, E. K., \& Shallice, T. Neuropsychological evidence of visual storage in short-term memory tasks. Quarterly Journal of Experimental Psychology, 1972, 24, 30-40.

Weiskrantz, L. A long-term view of short-term memory in psychology. In G. Horn \& R. A. Hinde (Eds.), Short-term changes in neural activity and behaviour. London: Cambridge University Press, 1970.

(Manuscript received April 27, 1983; revision accepted for publication September 20, 1983.) 